\title{
AN INTEGRATED APPROACH TO THE DEVELOPMENT OF CONTINUOUS SIMULATIONS
}

\author{
JAMES R. BURNS \\ Department of Systems, Texas Tech University, Lubbock, TX 79409, U.S.A.
}

and

ONUR ULGEN

Department of Industrial and Systems Engineering, University of Michigan-Dearborn, Dearborn, MI 48128, U.S.A.

\author{
(Received 1 September 1977; in revised form 18 April 1978)
}

\begin{abstract}
An integrated approach to the development of Forrester-style simulation models is described. The approach incorporates the concept of an interaction matrix to assist in the development of causal loop diagrams and Dynamo flow diagrams. The interaction matrix is derived from the fundamental notions of system dynamics. Premised upon the presumption that a computer-aided procedure for model formulation can expedite, systematize, and operationalize the model formulation process, the integrated approach utilizes the interaction matrix as a data structure within the computer. An algorithm designed to interface with a remote terminal (such as a CRT display) determines the interaction matrix by interrogating a user until sufficient information about the problem of interest has been obtained. This algorithm is also described in the paper. The interrogations both motivate and facilitate the determination of quantities to be included as well as couplings between the quantities. When a quantity or coupling is designated by a user, the algorithm "knows" its identity at the time of user origination. Both algorithm and matrix are illustrated through recourse to a text-book example and the paper concludes with a summarizing discussion of the possible contribution of such an approach.
\end{abstract}

\section{INTRODUCTION}

As governmental and industrial institutions find themselves embedded in problems which are increased in complexity and intensity, the need for more powerful techniques directed toward coping with these problems becomes ever more manifest. Simulation modeling is well established as a tool appropriate for the study and resolution of these problems. Even so, recent studies $[1,2]$ show that models often fall short of achieving their intended purposes. In a recent study funded by the National Science Foundation some 230 project directors responded to a mail survey intended to determine the uses and characteristics of currently extant models. It was determined that:

(1) "Not withstanding the great degree of policy intent, actual policy application appears to have the highest shortfall of use" ([1], p. 4).

(2) "The primary cause of low policy utilization rates for models probably is attributable to the "distance" between model builders and potential-policy makers ([1], p. 4).

This study furthermore stipulates that "at least one-third and perhaps as many as two-thirds of the models failed to achieve their avowed purposes in the form of direct application to policy problems". One is inclined to ask what can be done to improve the impact which models should be having upon actual policy application.

The study mentioned above was not intentioned as an assessment of the efficacy of current modeling methodology. However, from the statements above, it is clear that modeling methodologists should consider how changes in conventional methodology might mitigate the shortcomings alluded to. It is the thesis of this paper that, through computer-assistance, it would be possible to lessen the "distance" between model builders, analysts and policy makers. Such an approach could conceivably allow for policy maker participation in the model formulation exercise, could expedite the process of model development, making it less expensive while shortening model development time. Such shortened response periods might enable users to employ sophisticated models in crisis management situations.

The formulation of algorithms for this purpose forces a rigorous statement of the principles underlying the methodology. Specifically, the rules of system dynamics [6,7] are employed in the procedure to be discussed. Such algorithms would, when used, necessitate a systematic consideration on the user's part of the important aspects of the model structure. The approach would divide the labor of modeling into human and computer portions where the part not requiring human judgment is automated. The computer aids would also force the modeler to structure his model in a fashion consistent with the requirements of system dynamics methodology. Additionally, computer aids would allow for policy-maker participation in the model formulation process thereby increasing his understanding of model assumptions and structure while lessening model development costs. The policy-maker participatory methodologies of Kane [13] and Warfield [18] have already demonstrated their usefulness. If applied to simulation model-building, the approach might alleviate conventional problems of tedium, credibility and validity.

The general procedure of the approach taken herein is similar to a previous approach taken by Burns. Burns $[3,4]$ assumed that the causal loop diagram of the system was determinable through an extension of "structural modeling" techniques as developed by Warfield ([18], p. 345). Beginning with the causal loop diagram, Burns developed a technique for classifying the quantities and couplings into levels, rates, auxiliaries, etc., and thus to formulate the equations of the model. 
The procedure outlined in this paper will start not from the causal loop diagram but from scratch. Moreover, the approach taken herein is called the integrated approach because quantities, couplings, and their classifications are determined simultaneously. This is in contradistinction to the approach taken in $[3,4]$ which utilizes the separation conjecture of Warfield ([18], p. 63) first to list quantities, then to determine the couplings between the quantities and finally to classify couplings and quantities. (The separation principle avows that "greater intellectual productivity can be achieved by...conscious separation of mental activity into distinct idea actions...")

In addition to the integrated concept, the approach utilizes the notion of sector. A sector is a sub-structure of a causal loop diagram which contains all the structure directly associated with a material flow. Each sector contains one and only one material flow as shall be explained in later sections of the paper. The relationship of the notion of sector to the approach taken in [4] has been explored in [19]. The discussion in this paper concretizes and extends earlier results discussed in both [4] and [19].

Finally, in the approach to be presented, the possibility for computer-assistance in the formulation of system dynamics models is demonstrated. Structural information traditionally exhibited by means of the causal loop diagram (CLD) or the Dynamo fiow diagram (DFD) can be stored in the computer's memory by means of an "interaction matrix". Other similar model-building methodologies-see Kane[13], Wakeland[17], and Moll et al.[15]-expediciously utilize the notion of an interaction matrix. The use of interaction matrices in connection with system dynamics models was explored in [19]. Such matrices specify the interactions for all pairs $\left(q_{i}, q_{j}\right)$ defined on the Cartesian product $Q \times Q$, where $Q$ is a set of quantities.

In the next section the set-theoretical notions of system dynamics developed in $[3,4]$ will be reviewed in relation to their implications for the interaction matrix formally developed in [19]. In the section following, an algorithm for the integrated approach is described. The steps which comprise the algorithm are then illustrated through recourse to a textbook example. The specific queries which the algorithm would ask are indicated in the "example" section. The example is shown to faithfully conform to the format of the interaction matrix. In the last section, concluding assessments as to the relative contribution of the approach are given.

\section{ASSERTIONS OF SYSTEM DYNAMICS}

In previous papers $[4,19]$, the assertions of system dynamics have been reformulated using set and graph theory. In this section, those set-theoretic assertions will be restated in such a fashion as to allow for a nonrigorous heuristic rationalization of the interaction matrix. The interaction matrix has already been formally and rigorously derived [19].

The couplings adjacent to or associated with a quantity $q_{j}$ are designated by means of the sets $A_{c}\left(q_{j}\right), E_{c}\left(q_{j}\right)$, in which $A_{c}\left(q_{j}\right)$ denotes those couplings directed-toward $q_{i}$ (in the causal loop diagram) and $E_{\mathrm{c}}\left(q_{i}\right)$ denotes those couplings directed away-from $q_{j}$. From systems dynamics $[6,7]$, couplings represent either a transfer of substance (a flow, denoted $F$ ) or a transfer of in formation (denoted $I$ ). Definitions for these two coupling types appear later in this section.
Similar conventions are used for the quantities which are coupled to the quantity under consideration $q_{j}$. $E_{q}\left(q_{j}\right)$ denotes those quantities in $Q$ (the quantity set) which are coupled to $q_{i}$ by means of couplings directed away from $q_{j}$ (in the causal loop diagram) and $A_{q}\left(q_{j}\right)$ denotes those quantities which are coupled to $q_{i}$ by means of couplings directed toward $q_{j}$.

Set and logical symbols are also employed as follows. The notation $A_{c}\left(q_{j}\right) \subseteq I$, for example, denotes the proposition that $A_{c}\left(q_{j}\right)$ is a subset of the set $I$ of information couplings. When this proposition is simultaneous with the proposition $E_{c}\left(q_{j}\right) \subseteq I$ (denoting that the outward directed couplings are information couplings) then the compound proposition is written $A_{c}\left(q_{j}\right) \subseteq I \wedge E_{c}\left(q_{j}\right) \subseteq I$. Such propositions are used to define each of the five basic quantity types in system dynamics. These are stated below, in which $X$ denotes the set of states, $R$ denotes the set of rates, $V$ denotes the set of auxiliaries, $P U$ denotes the set of parameters and inputs, and $Y$ denotes the set of outputs.

States. A quantity is a state $\left(q_{i} \in X\right)$ if

$$
A_{c}\left(q_{i}\right) \subseteq F \wedge E_{c}\left(q_{i}\right) \subseteq I
$$

or if

$$
A_{q}\left(q_{i}\right) \subseteq R \wedge E_{q}\left(q_{i}\right) \subseteq V R Y .
$$

This definition asserts that (in the causal loop diagram) flow couplings are directed toward states and information couplings are directed away from states. In addition, states are affected only by rates, and states may affect auxiliaries, outputs, or other rates.

Rates. A quantity $q_{i}$ is a rate $\left(q_{i} \in R\right)$ if

$$
A_{c}\left(q_{i}\right) \subseteq I \wedge E_{c}\left(q_{i}\right) \subseteq F
$$

or if

$$
A_{q}\left(q_{i}\right) \subseteq P U V X \wedge E_{q}\left(q_{i}\right) \subseteq X
$$

This definition requires that (in the causal loop diagram) information couplings are directed toward rates and flow couplings are generally directed away from rates. Furthermore, rates may be affected by parameters, inputs, auxiliaries, or states, and rates may affect states.

The remaining definitions are given without justification.

Auxiliaries. A quantity is an auxiliary $\left(q_{i} \in V\right)$ if

$$
A_{c}\left(q_{i}\right) \subseteq I \wedge E_{c}\left(q_{i}\right) \subseteq I
$$

or if

$$
A_{q}\left(q_{i}\right) \subseteq P U V X \wedge E_{a}\left(q_{i}\right) \subseteq R V Y
$$

Parameters and inputs. A quantity is a parameter or an input $\left(q_{i} \in P U\right)$ if

$$
A_{c}\left(q_{i}\right)=\phi \wedge E_{c}\left(q_{i}\right) \subseteq I
$$

or if

$$
A_{q}\left(q_{i}\right)=\phi \wedge E_{q}\left(q_{i}\right) \subseteq R V Y
$$

Outputs. A quantity is an output $\left(q_{i} \in Y\right)$ if

$$
A_{c}\left(q_{i}\right) \subseteq I \wedge E_{c}\left(q_{i}\right)=\phi
$$


or if

$$
A_{q}\left(q_{i}\right) \subseteq P U V X \wedge E_{q}\left(q_{i}\right)=\phi
$$

Since each member of a particular quantity subset, such as $X, V$ or $R$, must by its definition, possess certain properties, all members of the quantity subset must possess the properties. Consequently, the following postulates represent restatements of the definitions given above.

Properties of the set of states $X$ :

$$
\begin{aligned}
& A_{c}(X) \subseteq F \wedge E_{c}(X) \subseteq I \\
& A_{q}(X) \subseteq R \wedge E_{q}(X) \subseteq V R Y .
\end{aligned}
$$

Properties of the set of rates $R$ :

$$
\begin{aligned}
& A_{c}(R) \subseteq I \wedge E_{c}(R) \subseteq F \\
& A_{q}(R) \subseteq P U V X \wedge E_{q}(R) \subseteq X
\end{aligned}
$$

Properties of the set of auxiliaries $V$ :

$$
\begin{aligned}
& A_{c}(V) \subseteq I \wedge E_{c}(V) \subseteq I, \\
& A_{q}(V) \subseteq P U V X \wedge E_{q}(V) \subseteq R V Y .
\end{aligned}
$$

Properties of the set of parameters and inputs $P U$ :

$$
\begin{gathered}
A_{c}(P U)=\phi \wedge E_{c}(P U) \subseteq I \\
A_{q}(P U)=\phi \wedge E_{q}(P U) \subseteq R V Y .
\end{gathered}
$$

Properties of the set of outputs $Y$ :

$$
\begin{aligned}
& A_{c}(Y) \subseteq I \wedge E_{c}(Y)=\phi \\
& A_{q}(Y) \subseteq P U V X \wedge E_{q}(Y)=\phi .
\end{aligned}
$$

In the postulates above, $A_{c}(X)$ denotes the entire set of couplings directed toward all states, while $E_{c}(X)$ denotes the entire set of couplings directed away from all states. The claim of postulate (1) is that couplings directed toward states are flow couplings, whereas couplings directed away from states are information couplings. This claim is substantiated by the first part of the definition for states. Since by definition each state possesses this property, all states possess the property. All of these definitions and properties are premised upon the consistency supposition [4]. A similar justification for the remaining nine properties is omitted here. See Ref.[19] for a thorough, formal derivation of these postulates.

The following definitions are given for flow and information couplings.

Flow couplings. Any coupling $c_{i j}=\left(q_{i}, q_{j}\right)$ whose $q_{i}$ is a rate or whose $q_{i}$ is a state, is a flow coupling; mathematically, this is written

$$
q_{i} \in R \vee q_{i} \in X \Rightarrow c_{i j} \in F
$$

Information couplings. Any coupling $c_{i j}=\left(q_{i}, q_{j}\right)$ whose $q_{i}$ is not a rate and whose $q_{j}$ is not a state is an information coupling; thus

$$
q_{i} \in P U V X \wedge q_{i} \in V R Y \Rightarrow c_{i j} \in I .
$$

In the sector approach the quantity set $Q$ is partitioned into sector quantities $Q_{i}$, and between-sector quantities $Q_{b}$. A sector will refer to a sub-structure (consisting of quantities and couplings) that can be associated with one and only one flow. Thus every flow defines a separate sector. All states and rates which either accumulate or control the flow are included within the sector. Auxiliaries are included within a sector only if they affect and are affected by other quantities known to be contained within a particular sector. Parameters and inputs are included only if they directly affect other quantities in the sector, while outputs are included within the sector if they are functions exclusively of quantities within that sector. Quantities which appear at the interface between two or more sectors are denoted by $Q_{b}=\left\{V_{b}, P U_{b}, Y_{b}\right\}$. $B y$ the definition of sector, between-sector quantities cannot include those quantities which control or accumulate flows, i.e. rates and states. All flows must occur within a sector and whenever a flow is observed, a sector must be defined for it. We define $Q_{b}$ as follows:

Between-sector quantities $\mathrm{Q}_{b} . \mathrm{Q}_{b}$ includes those quantities left over from the set subtraction $Q-\bigcup_{i=1}^{n} Q_{i}$ for a system of $n$ sectors.

\section{THE INTERACTION MATRIX}

With these brief concepts it is possible to introduce formally the interaction matrix. For simplicity the interaction matrix prescribing a two-sector system is considered, as shown in Fig. 1. below. Thus for a two-sector system, the interaction matrix can be partitioned into two sector matrices and two interconnection matrices. The interconnection matrices specify interactions between sectors, while the sector matrix specifies the interactions within a particular sector. Specifically, the $i \rightarrow j$ interconnection matrix specifies the interactions directed from sector $i$ toward sector $j$ and conversely for the $j \rightarrow i$ interconnection matrix. The sector and interconnection matrices are each discussed in what follows.

The sector matrix. The sector matrix is indexed by the sets $X, R, V, P U$ and $Y$ as shown in Fig. 2 below. The possible interactions in the sector matrix are indicated by a " $\pm I$ " or " $\pm F$ ", where " $\pm I$ " denotes possible information couplings, while " $\pm F$ " denotes possible flow couplings between members of the associated sets.

\begin{tabular}{l|l|l|}
\multicolumn{1}{c|}{ Sector $i$} & \multicolumn{1}{c|}{\begin{tabular}{c} 
Sector $i$ \\
Sector \\
\cline { 2 - 3 } Satrix $i$
\end{tabular}} & $\begin{array}{l}\text { Interconnection } j \\
\text { Matrix } i-j\end{array}$ \\
\cline { 2 - 3 } Sector $j$ & $\begin{array}{l}\text { Interconlection } \\
\text { Matrix } j \rightarrow i\end{array}$ & $\begin{array}{l}\text { Sector } \\
\text { Matrix } j\end{array}$ \\
\hline
\end{tabular}

Fig. 1. Interaction matrix for a two-sector system (no quantities between sectors are considered).

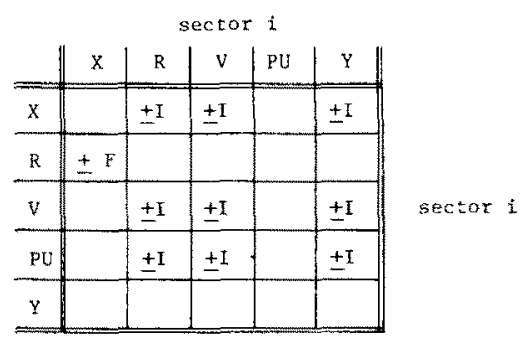

Fig. 2. Possible interactions in the sector matrix. 
Submatrices that are blank represent situations in which interactions cannot occur without producing a violation of the basic rules of system dynamics. For example, no entries can be placed in the $(X, X)$ or $(X, P U)$ submatrices because, according to the rules of system dynamics it is impossible for states to affect states directly or to affect parameters and inputs. The matrix can also be rationalized through a consideration of the set theoretic properties for rates, states, etc., provided earlier. By means of properties (1) and (2) above it is clear that states $X$ may affect only rates $R$, auxiliaries $V$, and outputs $Y$, and each by means of information transfers. This is depicted in the first row of the sector matrix shown in Fig. 2. Properties (1) and (2) also suggest that it is possible for states $X$ to be affected only by rates $R$, such affect being induced by means of flow couplings. This is depicted in the first column of the sector matrix. A similar consideration of the right-hand portions of properties $(3-10)$ for the remaining subset types justifies the remaining 4 rows of the sector matrix depicted above. An inspection of the columns reveals that the requirements of the left-hand sides of the properties are also satisfied.

The interconnection matrix. The interconnection matrix relating sector $i$ to sector $j$ is shown in Fig. 3 below. Clearly, many submatrices do not contain any interactions. By virtue of the definition of "sector", it is virtually impossible for quantities in $V, P U$ or $Y$ of sector $i$ to affect or be affected by quantities in $V, P U$ or $Y$ of sector $j$. Thus the submatrix indexed by $V, P U$ and $Y$ along both the row and column is entirely blank. It is also impossible for rates $R$ to affect any quantities in another sector without violating the definition of a sector or property (4) above. However, states $X$ may directly influence rates $R$ in another sector. States may not influence auxiliaries or outputs within another sector as those quantities must possess affector and effector subsets entirely contained within their respective sector(s). Thus the interconnection matrix allows only for interactions directed from states in sector $i$ to rates in sector $j$.

It is possible for some quantities to be incapable (by our conventions) of being associated with any one sector. Such quantities appear at the interface between two or more sectors and include auxiliaries $V_{b}$, parameters and inputs $P U_{b}$, and outputs $Y_{b}$. These between-sector quantities $Q_{b}$ must also be included in the interaction matrix in situations where they are required. The interaction matrix for two sectors with between-sector quantities included appears in Fig. 4.

Matrices labeled $\mathscr{A}$ in the interaction matrix depicted in Fig. 4 are simple sector matrices previously described, while matrices labeled $\mathscr{B}$ will be recognized as interconnection matrices. Matrix $\mathscr{B}$ is the matrix of information couplings directed from sector $i$ directly (i.e.

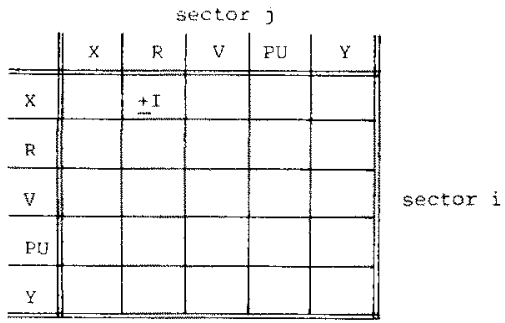

Fig. 3. Possible interactions in the interconnection matrix.

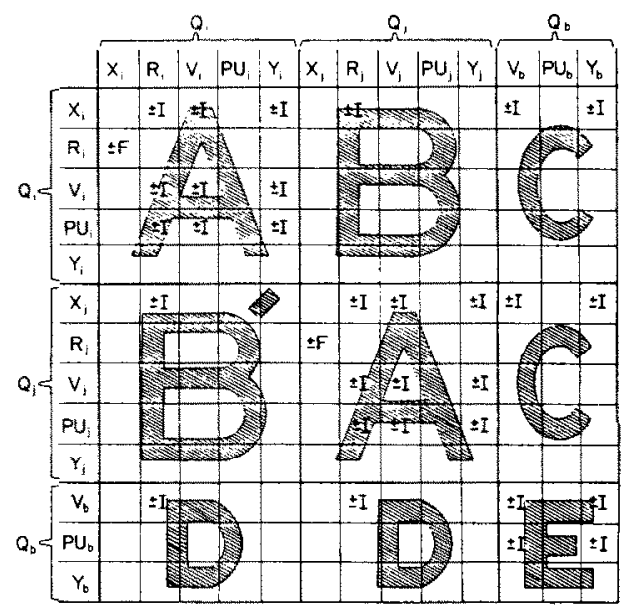

Fig. 4. Detailed interaction matrix for two-sector system (between sector quantities included).

there are no between-sector quantities) toward sector $i$, while matrix $B^{\prime}$ is the matrix of information couplings directed from sector $j$ directly toward sector $i$. Matrix $\mathscr{E}$ is the matrix exhibiting the possible interactions amongst the between-sector quantities. Matrices labeled $\mathscr{C}$ exhibit where and how quantities within sectors $i$ and $i$ can affect or influence between-sector quantities, while matrices labeled $\mathscr{D}$ exhibit where and how between-sector quantities can influence quantities within sectors $i$ and $j$. Matrices $\mathscr{C}$ and $\mathscr{D}$ could also be thought of as interconnection matrices, but to avoid possible confusion with matrices $\mathscr{B}$, these matrices shall be referred to as interface matrices. Note that the only matrices in which flow couplings can occur are in the sector matrices labeled $\mathscr{A}$-all remaining matrices contain exclusively information couplings.

If the interaction matrix were used in a computerassisted model formulation exercise, an interrogation would be performed by the computer for each of the entries in which a possible interaction could take place. Using the interaction matrix as its data structure, the computer would insist upon a comprehensive consideration of all feasible interactions that are consistent with the rules of system dynamics. A thorough justification of the format of the interaction matrix is found elsewhere[19].

\section{THE ALGORTHM}

The algorithm embodying the integrated approach is broken into phases which are illustrated and described by what follows. Employed in an interactive time-sharing, computational mode, the algorithm interrogates a user or panel of users until sufficient information about the issue of interest has been obtained. The sequence of interrogations are intended to both motivate and facilitate the development of the model structure. The responses resulting from the sequence of interrogations would be stored in the data structure prescribed by the interaction matrix. The algorithm assumes that its users can identify the important quantities and interactions in the system.

The algorithm forces the user to proceed in a systematic manner, naming sectors and quantities and specifying relationships between sectors and between quantities. The algorithm is comprised of the following phases. 
Phase A. Model builders identify the sectors (subsystems) of the system and consider the agregate interactions among the sectors. Later phases may require the introduction and/or elimination of one or more sectors. A comparison of the initial intuitive sector model with the final model will point out the directions taken with respect to the assumptions, level of detail, and components of the system. A DELTA chart of Phase A is depicted in Fig. 5.

Phase B. Since the states of a system are easiest to identify, especially after the sectors of the same are specified, this phase considers first the state variables of each sector. Then the rates that control each level variable are identified and for each sector a state-rate interaction MSTM $\dagger$ and DFD is constructed, in accordance with the DELTA chart in Fig. 6 below, where $A_{R_{i}}\left(X_{i}\right)$ denotes those rates in sector $i$ which directly affect the

† Modified Square Ternary Matrix $[4,19]$.

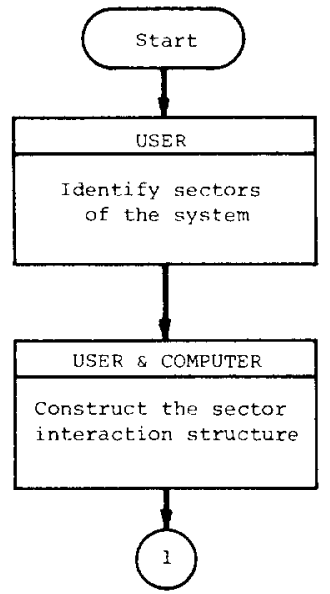

Fig. 5. DELTA chart of Phase A.

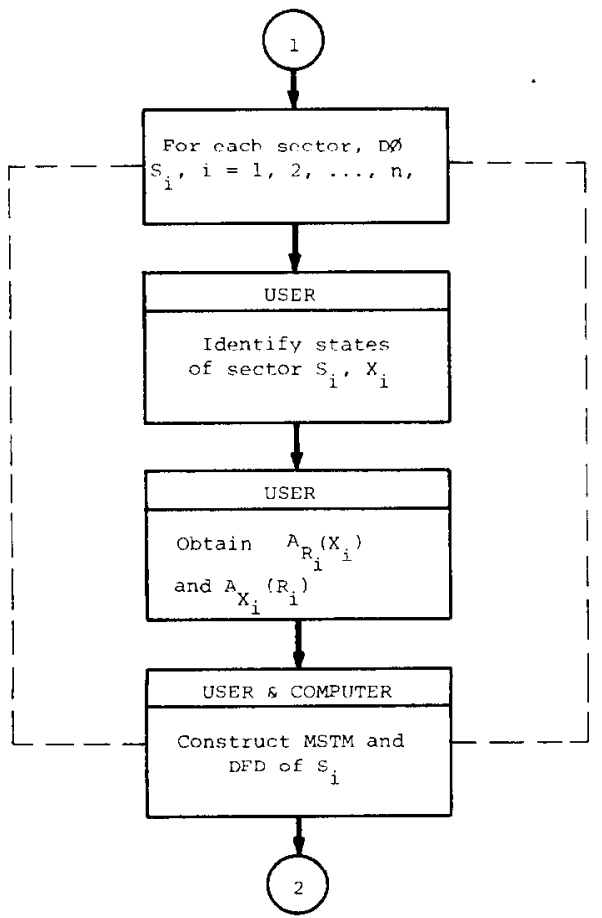

Fig. 6. DELTA chart of Phase B. set of states in sector $i$, and conversely for $A_{X_{i}}\left(R_{i}\right)$. At the last step of Fig. 6, in constructing the MSTM of the system sectors $S_{i}$, the user identifies the sign of interactions $c_{i j}$ as positive or negative between the withinsector states and rates by responding to the queries asked by the computer. The specific queries posed by the algorithm are derived from the information supplied by the user during previous steps and by the possible interactions as dictated by the interaction matrix.

Phase $C$. In this phase the algorithm asks queries to obtain the interaction structure between the states of one sector and the rates of other sectors. The type of interaction, direct or indirect, is not considered at this stage. The DELTA chart of this phase, depicted in Fig. 7, uses the notation $A_{X_{j}}\left(R_{i}\right)$ to denote states in sector $j$ which affect, directly or indirectly, rates in sector $i$. In this phase the user is asked to respond to a query of the form, "Does $x_{l}$ affect $r_{k}$ either directly or indirectly?"

Phase $D$. The type of interaction as direct or indirect is determined and used at this phase to identify the between-sector quantities, if any. The between-sector state-rate interactions designated in the previous phase are re-examined to determine if there are intervening auxiliaries along the path-such an effect we term "indirect". Direct and indirect interactions of $\left(x_{i}, r_{k}\right)$ are simultaneously possible in system dynamics. In considering the indirect interactions one starts building up the between-sector quantity set. Figure 8 DELTA chart delineates the key role the auxiliary variables play at this phase of the algorithm. User-computer interaction generates the between-sector quantity structure by identifying the affector and effector sets of the betweensector auxiliary variables. From eqn (6) effector sets of auxiliary variables may be rates, outputs, and other auxiliaries $\left(E_{R}\left(V_{b}\right), E_{Y}\left(V_{b}\right), \quad E_{V_{b}}\left(V_{b}\right)\right) . \quad$ Similarly, affector sets of auxiliary variables may include parameters, inputs, states and other auxiliaries $\left(A_{P_{b}}\left(V_{b}\right), A_{U_{b}}\left(V_{b}\right), A_{X}\left(V_{b}\right), A_{V_{b}}\left(V_{b}\right)\right)$. All the input, parameter, output and auxiliary variables identified in this phase belong to the between-sector quantity set $Q_{b}=V_{b} P_{b} U_{b} Y_{b}$. If a new rate and/or state variable is identified at this phase, a new sector must be defined for it, necessitating a return to Phase $A$, in which the new sector and its interactions with other sectors are specified (not shown in Fig. 8).

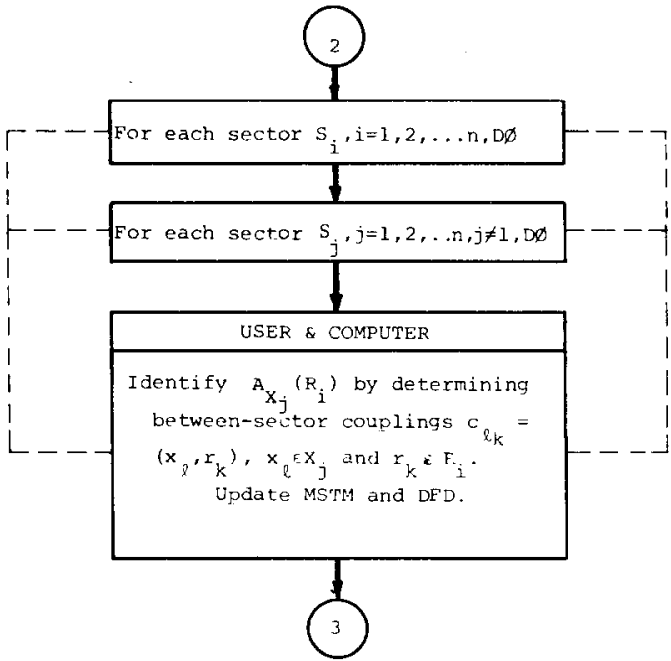

Fig. 7. DELTA chart of Phase C. 


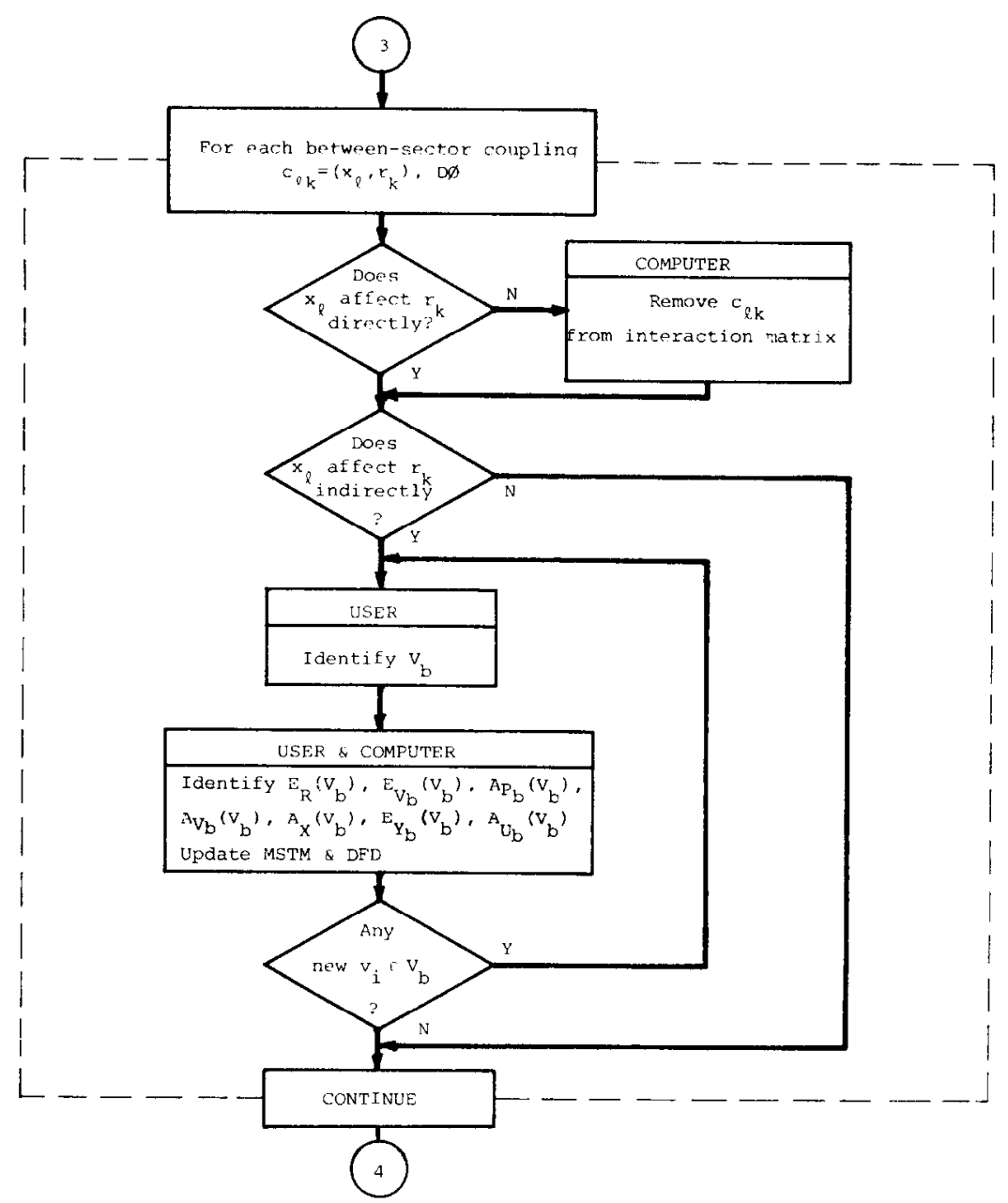

Fig. 8. DELTA chart of Phase D.

Phase $E$. In this last phase the affector sets of the rate variables of each sector are identified. From eqn (4) the affector sets of rate variables may be parameters, inputs, auxiliaries and states. The purpose of this phase is to develop the necessary supportive structure within each sector. For each sector, all rates within the sector are inspected for possible supportive parameters and auxiliaries. The user inputs the parameters and auxiliaries through the terminal. For each newly inputted auxiliary $v_{i}$, the algorithm asks the user to identify existing affector and effector quantities (represented by $E_{R}\left(v_{i}\right)$, $E_{V}\left(v_{i}\right), E_{Y}\left(v_{i}\right), A_{p}\left(v_{i}\right), A_{U}\left(v_{i}\right), A_{V}\left(v_{i}\right)$ and $\left.A_{X}\left(v_{i}\right)\right)$ to which $v_{i}$ must connect. The MSTM within the computer's memory is then appropriately updated. The DELT A chart for this phase is depicted in Fig. 9.

In the next section, each of these phases is further broken down into "steps" and illustrated by means of recourse to a typical textbook example.

\section{AN APPUCATION OF THE INTEGRATED} APPROACH

This section illustrates the concepts of the integrated approach through the use of an example problem. The problem chosen for this purpose is an adapted version of the residential community model discussed on pp.309-347 of [11] and patterned after Urban Dynamics[8]. The problem addressed by the model is the dynamic inter- action between population growth and available housing in a residential community such as a resort town. The description of the problem is given below as part of the first step of the integrated approach. The general steps to an integrated approach were discussed by the authors elsewhere [19] but a specific algorithm with its particular order and form of queries was not considered. The algorithmatized integrated approach consists of the following steps:

(1) Familiarization with the problem and approach.

(2) Determinization of the sectors that comprise the system.

(3) Determination of interaction among sectors.

(4) Determination of state-rate interactions within sectors.

(5) Determination of interaction state-rate pairs between sectors.

(6) Determination of between-sector quantities.

(7) Determination of within-sector parameters, inputs, outputs and auxiliaries.

(8) Insertion of delays where appropriate.

The above steps will achieve complete specification of the interaction matrix from which the Dynamo flow diagram of the problem can easily be delineated. Note that Steps 2 and 3 complete Phase $A$ of the algorithm, Step 4 accomplishes Phase B, Step 5 accomplishes Phase C, Step 6 completes Phase D and Step 7 completes Phase 


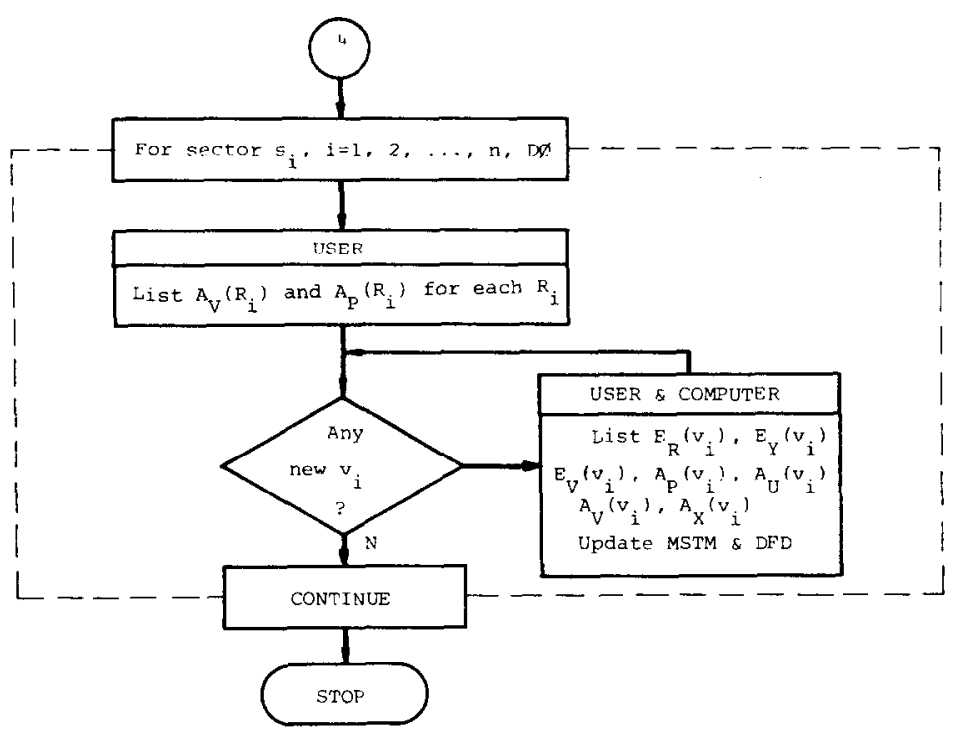

Fig. 9. DELTA chart of Phase E.

E. An iteration through the steps of the integrated approach yields the following results.

Step 1: Familiarization with the problem and approach. The residential community considered is a resort town within a fixed geographical area. The population growth in the area depends on the availability of housing as well as the steady natural attractiveness of the area. Natural attractiveness of the area brings people at a certain rate depending on the resident population level as long as the desire for housing matches the availability of houses. Abundant housing attracts people at a greater rate than under normal conditions. The opposite is true when the housing situation is tight. Area residents also leave the community at a certain rate due to various reasons. This outflow of residents also depends on the housing situation.

Housing construction industry, on the other hand, fluctuates depending on the land availability and housing desires. Abundant housing cuts back the construction of houses while the opposite is true when the housing situation is tight. Also, as land for residential development fills up, the construction rate decreases down to the level of demolition rate of houses.

†All user-computer conversations depicted in Fig. 10 and following come from an actual interactive user-computer exercise. The software employed is documented by Refs. [20] and [21].
Step 2: Determination of the sectors that comprise the system. Figure 10 is an illustration of how the user(s) can converse with the computer program (algorithm) through simple responses to questions from the program. The user answers queries with the information requested. Two sectors or subsystems are identified for the residential community. They are housing $\left(S_{1}\right)$ and population $\left(S_{2}\right)$. These two sectors delineate a rough boundary for the system considered.

Step 3: Determination of interaction among sectors. Both sectors affect each other since there is a close relationship between people living in an area and the housing available in the area. There exists a positive feedback loop between the subsystems as shown in Fig. 11. In other words, the housing sector stimulates a larger population sector which further stimulates the housing sector. This structure was elicited from the user by the queries depicted in Fig. 10 below. $\dagger$

Step 4: Determination of state-rate interactions within sectors. For the housing sector, there is a flow involving houses from new construction to demolition. The number of houses in the area at any time can be accumulated in one level or state variable called simply housing and represented by $x_{1}$. Symbolically, $X_{1}=$ $\left\{x_{1}\right.$ : housing $\}$. The rate variables, $R_{1}$, that control the flow of houses into and out-of the housing state variable $x_{1}$ are new housing construction rate $\left(r_{1}\right)$ and housing demolition rate $\left(r_{2}\right)$ respectively. In set notation, $R_{1}$ : the set of rates in sector $S_{1}=\left\{r_{1}\right.$ : new construction rate, $r_{2}$ :

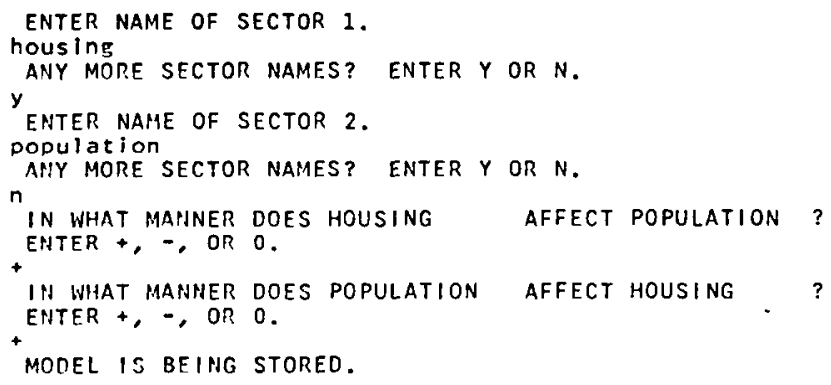

Fig. 10. Conversation between computer and user at Phase A for the residential community model. 


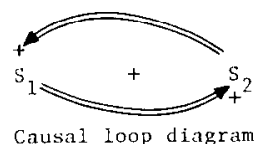

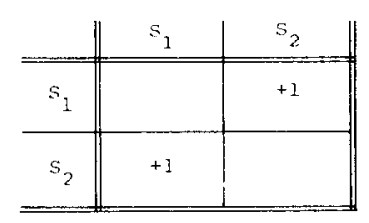

Square ternary matrix
Fig. 11. Interactions between population and housing sectors.

demolition rate $\}$. Since both of these rates are increasing or decreasing the housing stock as a certain percentage of the number of houses each year, the state (housing) affects both of the rate variables. These interactions among the states and rates of the housing sector lead to the Dynamo flow diagram. The actual queries asked by the computer during the modeling exercise follow the steps of the Phase B DELTA chart of Fig. 6. A portion of the conversation is delineated in Fig. 13. As each rate is associated with a state, the sign of the coupling is inserted and the current list of the rates is updated for correct state-rate interaction structure within each sector.

In considering the demographic or population sector, there is a flow involving people. The number of persons living in the community at any time can be accumulated in one level or state variable. The population state variable is represented by $x_{2}$. Thus $X_{2}=\left\{x_{2}\right.$ : population $\}$. The rates of demographic sector, $R_{2}$, that control the level of population are the in-migration to the area $\left(r_{3}\right)$,

In the actual computer conversation itself these names were abbreviated to "home cons rte" and "home demo rt" because of a twelve-character name-length limitation in the program. out-migration from the area $\left(r_{4}\right)$ and the net death rate $\left(r_{5}\right)$ in the area. The population experiences an annual net death rate due to the largely elderly character of the local population. Symbolically, $\boldsymbol{R}_{2}=\left\{r_{3}\right.$ : in-migration rate, $r_{4}$ : out-migration rate, $r_{5}$ : net death rate\}. These are abbreviated "in-migr rate", "out-migr rte" and "net-death $\mathrm{rt}$ " in conversations to follow.

The interactions among the states and the rates of the demographic sector are specified next. Since the rates of this sector increase or decrease the population level as a certain percentage of the population at any time, all the rates of this sector are affected by the population state variable. The MSTM and DFD depicted in Fig. 14 characterize the state-rate interactions for the demographic sector.

Step 5: Determination of interacting state-rate pairs between sectors. In Step 3, the interactions between sectors were specified. Generally, all sectors of interest must be coupled with at least one of the other sectors of the system; otherwise, isolated sectors can be investigated separately. The only type of interaction between sectors is the cross-sector state-rate interaction via an information link. This is prescribed by the interconnection matrix of Fig. 4.

From the problem description, housing $x_{1}$ affects the rates of in-migration $r_{3}$ and out-migration $r_{4}$. Similarly, population $x_{2}$ has an effect on the housing construction rate $r_{1}$. Consequently, there are information links in the $\left(x_{1}, r_{3}\right),\left(x_{1}, r_{4}\right)$ and $\left(x_{2}, r_{1}\right)$ positions of the MSTM. Note that at this point there is little concern about whether the cross-sector information links are direct or indirect as this issue will be taken up in the next step. Figure 15 exhibits one possible format for the queries of this step. Queries requesting the sign of the paths are not
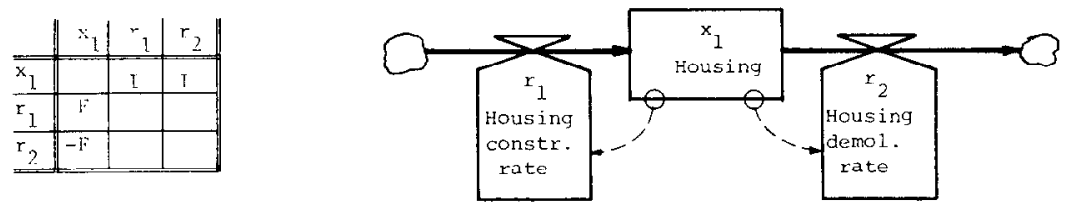

State-rate MSTM of sector 1

State-rate DFD of sector 1

Fig. 12. State-rate interactions within housing sector.

CONSIDER SECTOR 1: HOUSING

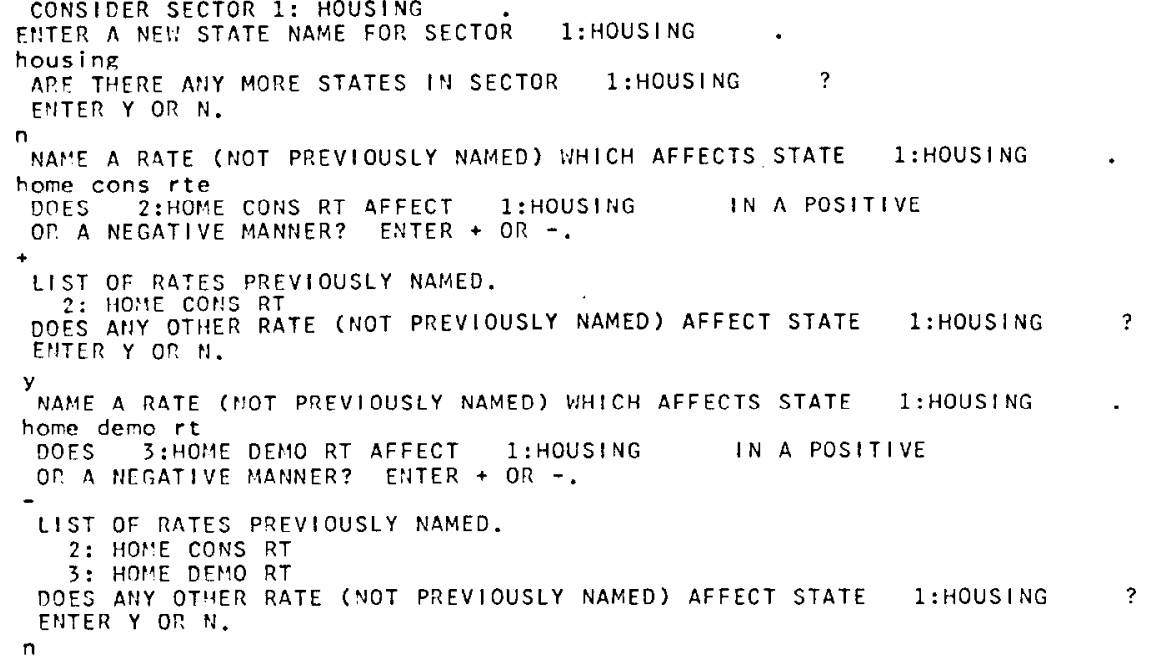

Fig. 13. A portion of Phase B (Step 4) conversations for the residential community problem. 

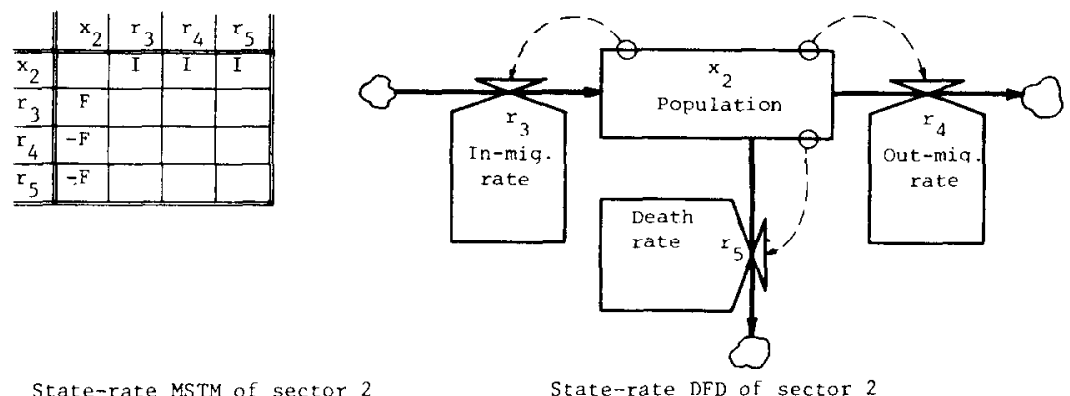

State-rate MSTM of sector 2

State-rate DFD of sector 2

Fig. 14. State-rate interactions within demographic sector.

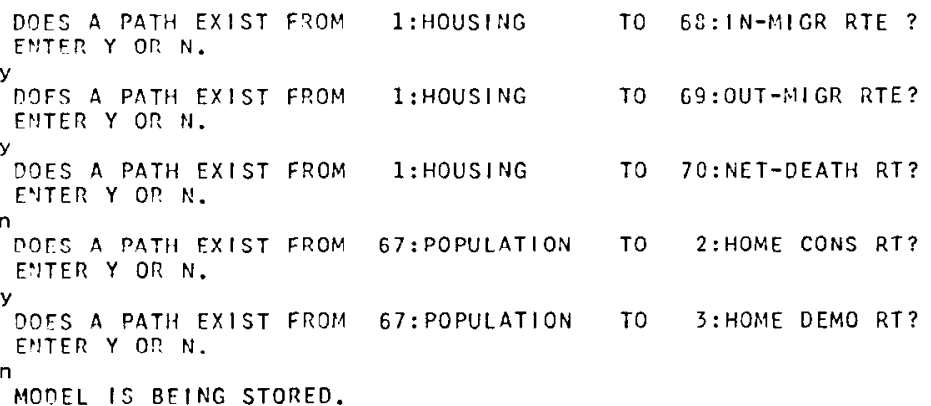

Fig. 15. Conversations related to Phase $\mathrm{C}$ (Step S) for the residential community model.

necessary at this juncture; later when the specific couplings which comprise a path are determined, the sign of these couplings will also be determined.

Step 6: Determination of between-sector quantities (auxiliaries, outputs, parameters and inputs). The previous step disclosed three between-sector information paths: $\left(x_{1}, r_{3}\right),\left(x_{1}, r_{4}\right)$ and $\left(x_{2}, r_{1}\right)$. In this step of the sector approach the between-sector information paths are each considered separately with the intent of determining if intermittent auxiliaries with adjacent inputs parameters, or outputs are part of the information path. Should this step generate between-sector quantities that are believed to be of types other than auxiliaries, parameters or inputs (such as rates or states), then a new sector will have been identified and the user must return to Step 3. Each of the linkages found in Step 5 are considered separately in the following discussion.

Information link $\left(x_{1}, r_{3}\right)$ : Effect of housing $x_{1}$ on inmigration rate $r_{3}$ is indirect through auxiliary variables that specify the condition of the housing market in the area. One quantity which is important is the housing ratio $v_{1}$, which is defined as the ratio of housing level $x_{1}$ to housing desired at any time. As available housing begins to exceed housing desired, housing ratio becomes greater than one and the area becomes more attractive for in-migration. The opposite is true when the housing ratio becomes less than one. Since the area attracts a certain in-migration under normal conditions (when $v_{1}=1$ ), housing ratio influences another auxiliary variable (a table function) called attractiveness for in-migration multiplier $v_{2}$ which in turn increases or decreases the in-migration rate depending on the housing ratio. Thus, between-sector quantities are discovered along the information link directed from housing $x_{1}$ to in-migration rate $r_{3}$ and include the between-sector auxiliary variables housing ratio $v_{1}$ and attractiveness for migration multiplier $v_{2}$. Symbolically, $V_{b}=\left\{v_{1}, v_{2}\right\}$. The character of the queries of this portion of the algorithm is exhibited in Figure 16. The queries enable the determination of direct and indirect linkages, which may coexist in parallel. And, queries within this phase also determine the sign of each coupling.

Let us now consider the quantities in the affector and effector sets of these in-between auxiliaries. Housing ratio $v_{1}$ is affected by housing $x_{1}$ and housing desired $v_{3}$. Housing desired $v_{3}$ is a quantity which depends on the current population $x_{2}$ living in the area and a parameter that specifies housing units required per person $p_{1}$. Attractiveness for migration multiplier $v_{2}$, on the other hand, is influenced only by the housing ratio $v_{1}$. Symbolically, $V_{b}=\left\{v_{1}\right.$ : housing ratio, $v_{2}$ : attractiveness for migration multplier, $v_{3}$ : housing desired $\}$ and $P U_{b}=\left\{p_{1}\right.$ : housing units required per person\}. This structure is depicted in Fig. 17(b).

Information link $\left(x_{1}, r_{4}\right)$ : Effect of housing $x_{1}$ on outmigration rate $r_{4}$ is again indirect and similar in many ways to the $\left(x_{1}, r_{3}\right)$ linkage. Attractiveness of migration multiplier $v_{2}$ which affects the in-migration rate $r_{3}$ can be thought of as affecting the out-migration rate $r_{4}$ if its inverse is taken. The inverse of the attractiveness multiplier, called the departure migration multiplier $v_{4}$, regulates the out-migration rate. In other words, as the area becomes more attractive due to favorable effect of housing ratio, more people arrive and fewer people depart than normally. Consideration of the $\left(x_{1}, r_{4}\right)$ linkage added one more between-sector auxiliary variable, the departure migration multiplier $v_{4}$, to the set of between-sector quantities so that $Q_{b}=V_{b} P_{b} U_{b} V_{b}=$ $\left\{v_{1}, v_{2}, v_{3}, v_{4}, p_{1}\right\}$.

Information link $\left(x_{2}, r_{1}\right)$ : The effect of population $x_{2}$ on housing construction rate $r_{1}$ is indirect too. As the housing desired increases as a function of population, housing ratio decreases and the high demand on houses makes it more attractive for construction. Housing con- 


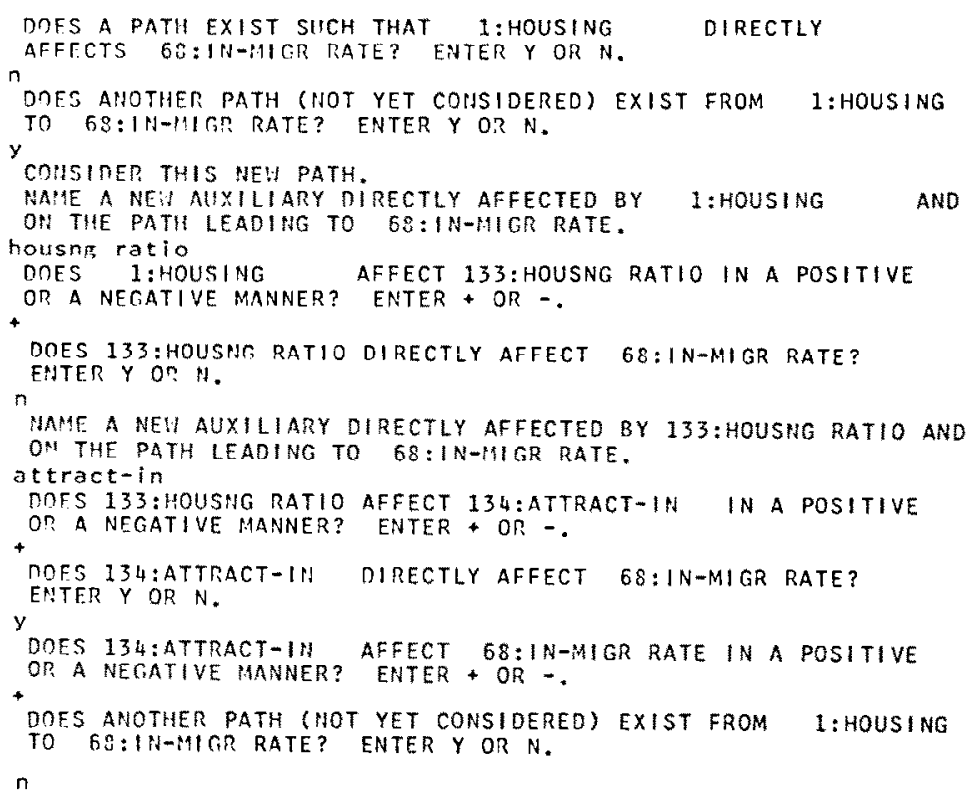

Fig. 16. A portion of phase D conversations for the residential community model.

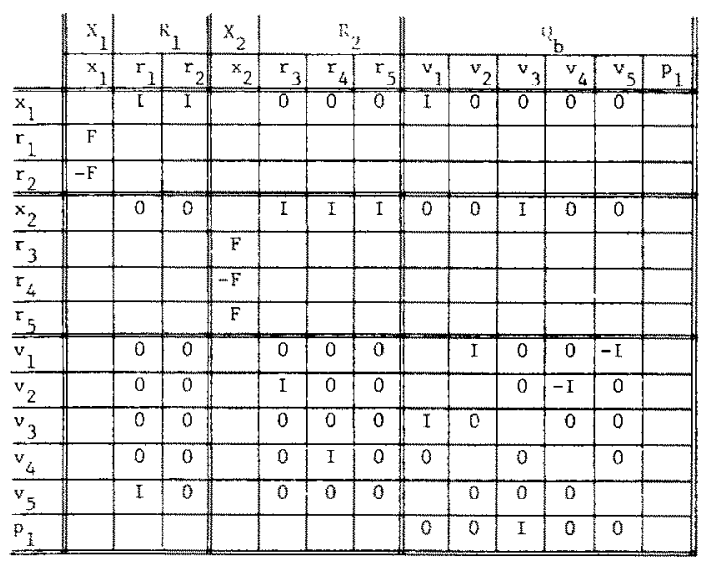

Fig. 17(a). System MSTM incorporating $X, R$ and $Q_{b}$, but not $V$, $P U$ within the two sectors.

struction multiplier $v_{5}$ which adjusts the normal construction rate upward or downward to reflect housing market conditions then is an additional between-sector auxiliary variable (a table function) that links the housing construction rate $r_{1}$ to housing ratio $v_{1}$.

The system MSTM and DFD incorporating the between-sector quantities is shown in Fig. 17. In the interaction matrix of Fig. 17 blank entries refer to inferred non-interactions. Zero entries represent situations where non-zero entries could have been inserted without violating the rules of system dynamics but were not because they do not characterize the issue at hand. It is observed that all linkages for the model do occur in those submatrices where, according to the interaction matrix depicted in Fig. 4, linkages can occur.

Step 7: Determination of within-sector parameters, inputs, outputs and auxiliaries. In this step the necessary support structure within each sector is determined. We proceed by identifying the affector sets of the sector rates. Only auxiliaries, parameters and inputs are brought into the model at this stage and they are further in- vestigated with respect to their interaction with the other quantities of the sector.

Within the housing sector $S_{1}$, there are two rates pertinent to the sector-housing construction rate $r_{1}$ and housing demolition rate $r_{2}$. Availability of land is considered of major importance for the housing sector. Housing construction rate $r_{1}$ is affected by the land fraction occupied in such a way that as residential development areas fill up, the construction rate decreases. As long as the land fraction occupied is low, housing construction rate will follow the normal rate. As a result, we can identify land fraction occupied $v_{6}$ affecting the housing construction rate $r_{1}$ through a multiplier called the land availability multiplier $v_{7}$. A parameter called normal housing construction $p_{2}$ specifies the construction rate under normal conditions. Land fraction occupied $v_{6}$ is affected by the total residential land $p_{3}$, land occupied by each house unit $p_{4}$, and the level of housing $x_{1}$. Queries transmitted by this portion of the algorithm are depicted in Fig. 18. The selected computer conversation is characteristic of the integrated approach where the type and sign of couplings as well as the type of quantities are generated simultaneously. The algorithm also interrogates the user on the existence of alternate paths among quantities. Throughout the modeling exercise, the user has access to the current listings of the affector and effector sets of a given quantity as well as to the list of candidate quantities which may potentially affect or be affected by a given quantity.

In considering the other rate of the housing sector, housing demolition rate $r_{2}$ simply depends on average lifetime of housing $p_{5}$. The manner in which withinsector quantities of sector $S_{1}$ interact with other quantities is prescribed by the sector matrix, Fig. 2. For this sector involving 81 pairs, interactions between 54 of them are not possible $\mathrm{A}$ consideration of the remaining 27 (non-blank) pairs leads to the interactions indicated in Fig. 19, where the associated flow diagram is also shown. This completes the structural description for the housing sector $S_{1}$.

For the population sector $S_{2}$ we have identified three 


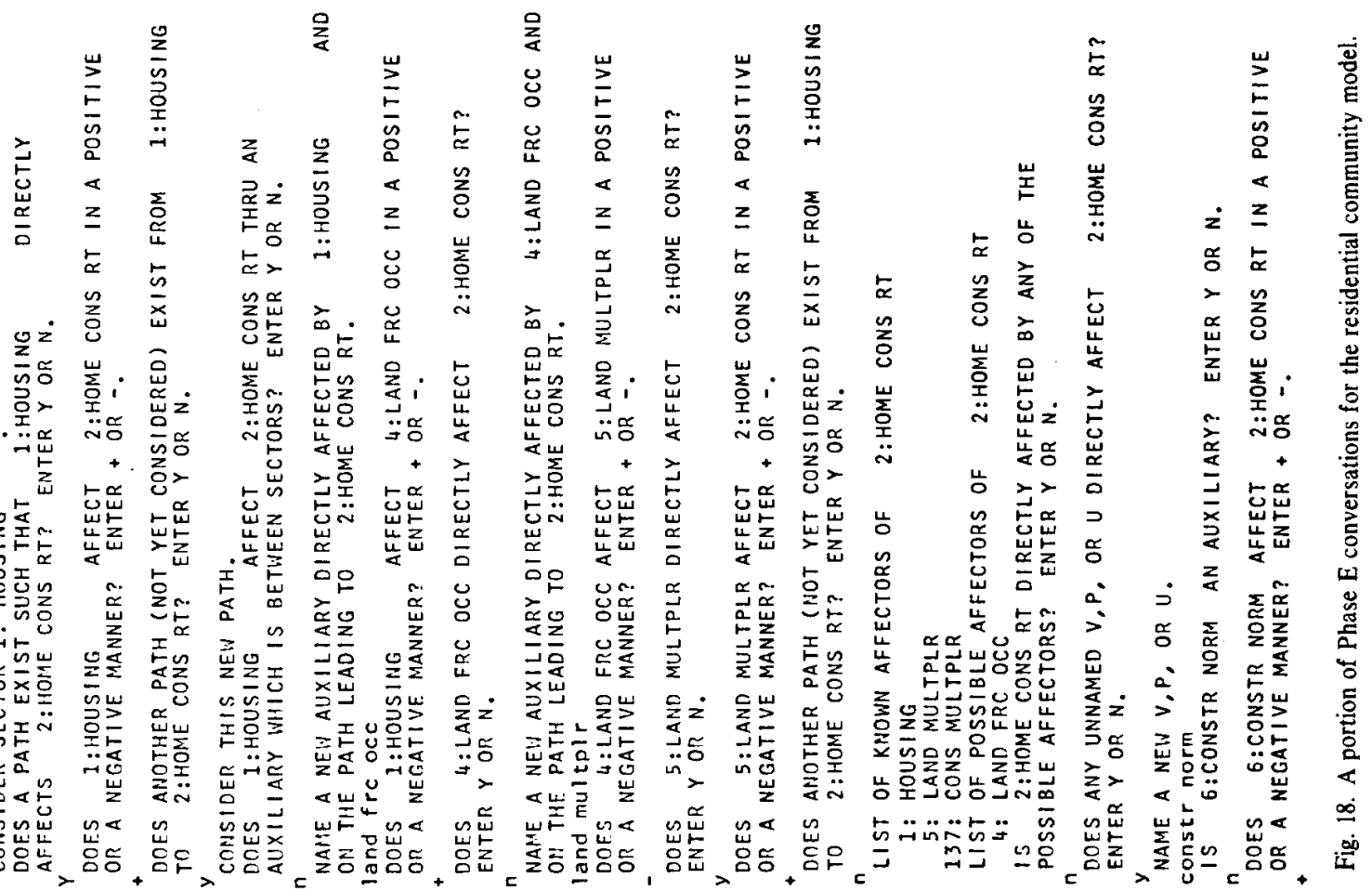

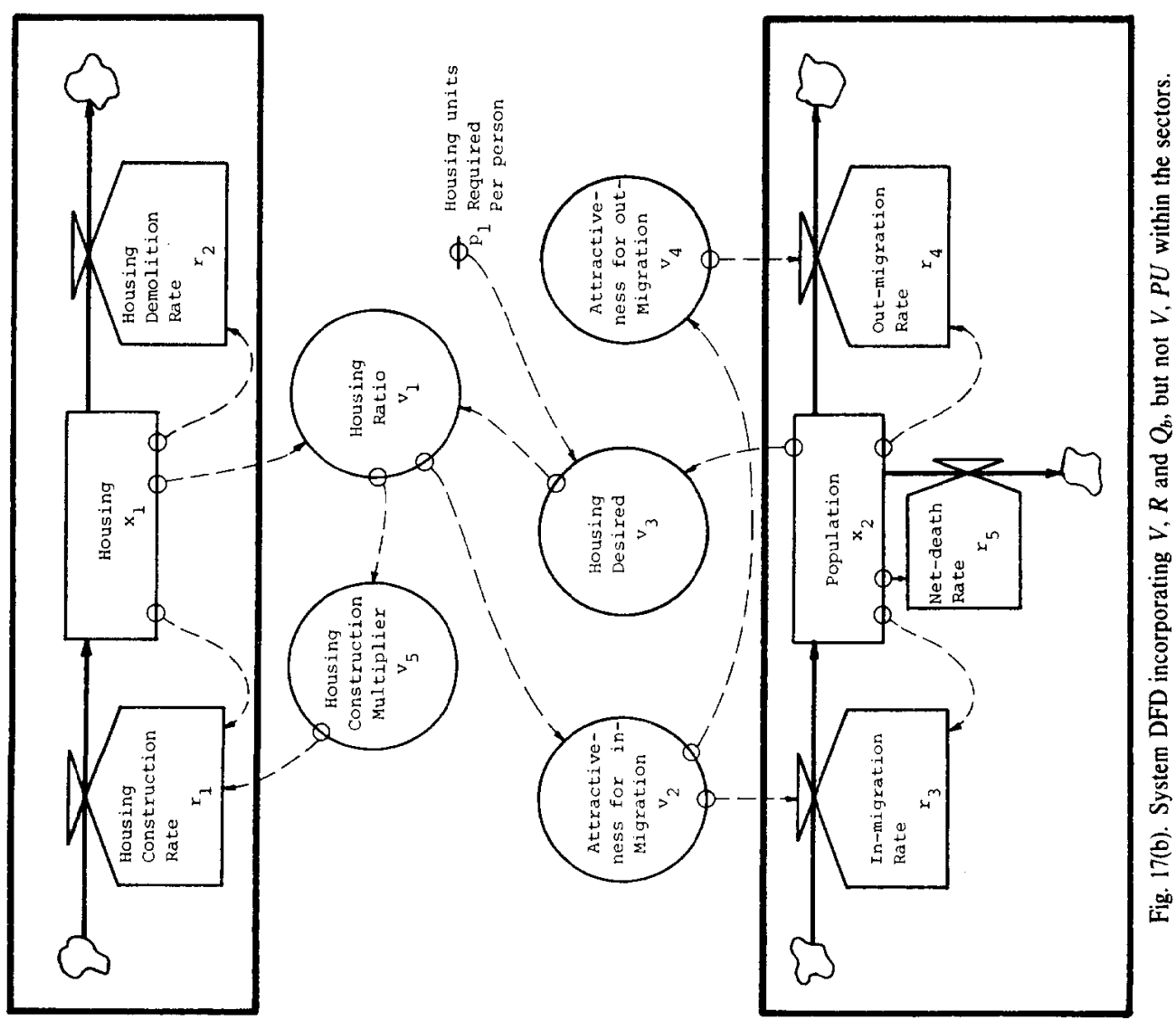



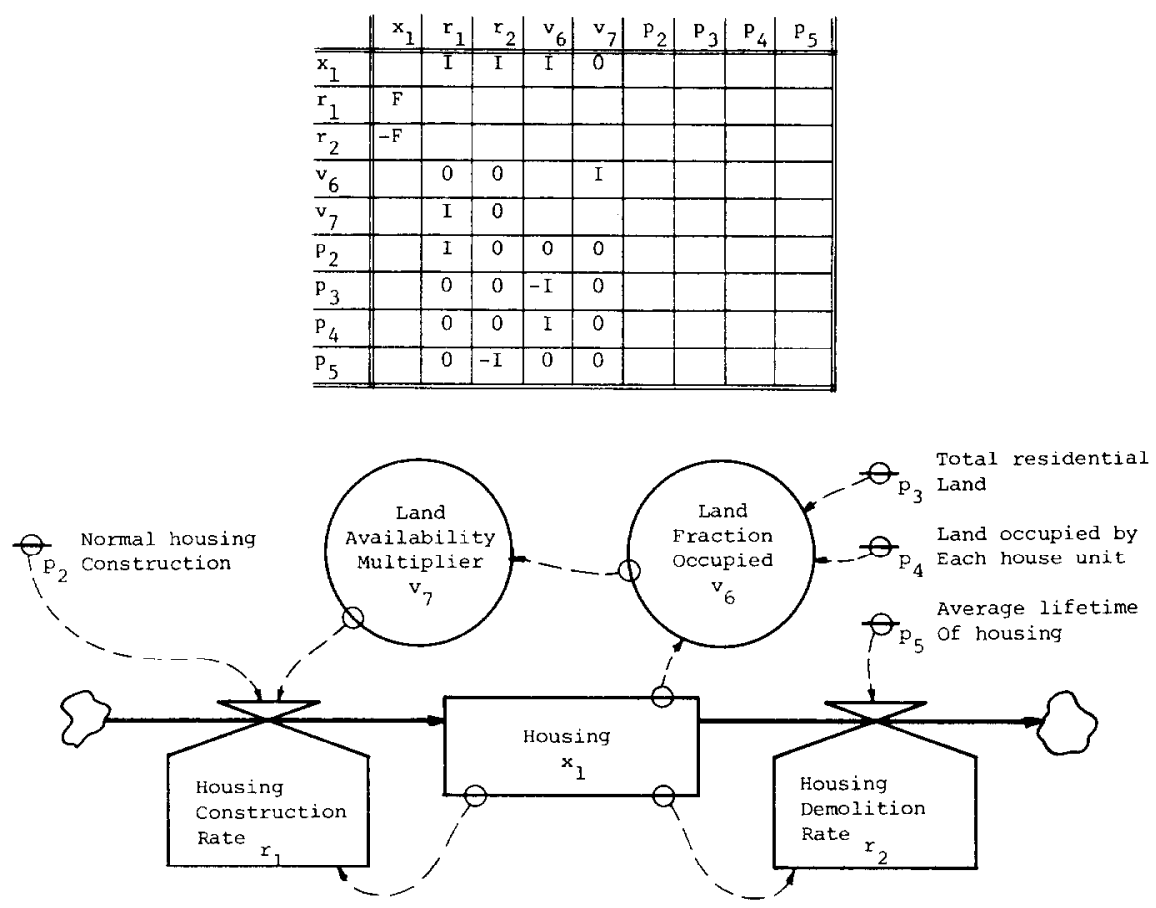

Fig. 19. Structural description for the housing sector, including $V, P U$.

rates; namely, in-migration rate $r_{3}$, out-migration rate $r_{4}$, and the net death rate $r_{5}$. Normal in-migration $p_{6}$ and normal out-migration $p_{7}$ are the parameters affecting in-migration rate and out-migration rate under normal conditions-i.e. when housing desired equals the housing available. Similarly, the net death rate at the community depends on another parameter, death rate factor $p_{8}$, which is constant due to the age structure of the residential community population. The interactions are determined from a consideration of the sector matrix, Fig. 2. The resultant matrix and associated DFD are shown in Fig. 20. For this sector, 34 of the 49 possible pairs are inferred as noninteractant (all which are blank in Fig. 20). Thus only 15 pairs (those which are nonblank) permit interactions consistent with the rules of system dynamics.

The computer would utilize the previously obtained information about the problem of interest to complete the interaction matrix depicted in Fig. 4. The resultant interaction matrix for the example problem is shown in Fig. 21 together with the associated fiow diagram. Blank entries were inferred, while zero entries represent situations where interactions were feasible but nonetheless not representative of the problem considered.

Step 8: Insertion of delays where appropriate. An examination of information links in the models shows the
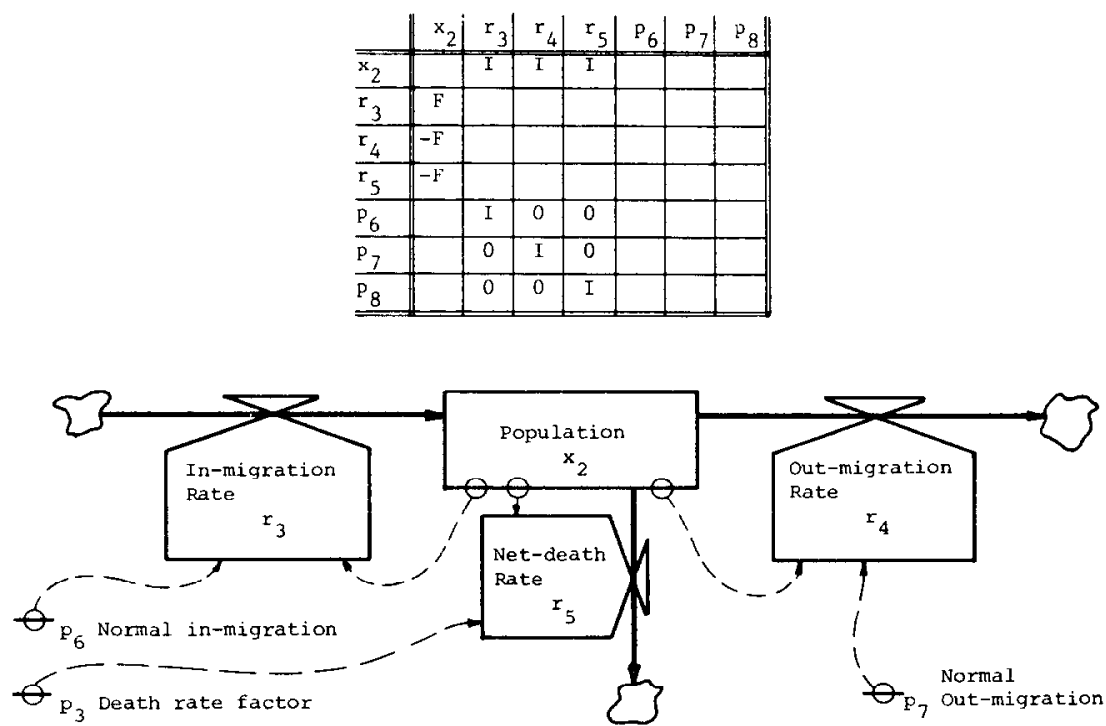

Fig. 20. Structural description for the demographic sector, including $V, P U$. 
40

\begin{tabular}{|c|c|c|c|c|c|c|c|c|c|c|c|c|c|c|c|c|c|c|c|c|c|c|}
\hline & \multicolumn{9}{|c|}{$Q_{1}$} & \multicolumn{7}{|c|}{$Q_{2}$} & \multicolumn{6}{|c|}{$Q_{b}$} \\
\hline & $x_{1}$ & \multicolumn{2}{|c|}{$\mathrm{R}_{1}$} & \multicolumn{2}{|c|}{$v_{1}$} & \multicolumn{4}{|c|}{$\mathrm{P}_{1}$} & $\mathrm{x}_{2}$ & \multicolumn{3}{|c|}{$\mathrm{R}_{2}$} & \multicolumn{3}{|c|}{$\mathrm{P}_{2}$} & \multicolumn{5}{|c|}{$v_{b}$} & $P_{b}$ \\
\hline & ${ }^{x} 1$ & $I_{1}$ & ${ }^{5} 2$ & $\mathrm{v}_{6}$ & $v_{7}$ & $\mathrm{P}_{2}$ & $\mathrm{P}_{3}$ & $P_{4}$ & $\mathrm{P}_{5}$ & $\mathrm{x}_{2}$ & ${ }^{\mathrm{r}} 3$ & $r_{4}$ & ${ }^{{ }^{r}} 5$ & $P_{6}$ & $P_{7}$ & $\mathrm{P}_{8}$ & $v_{1}$ & $v_{2}$ & $\mathrm{v}_{3}$ & $v_{4}$ & $v_{5}$ & $\mathrm{p}_{1}$ \\
\hline $\mathrm{x}_{1}$ & & I & I & I & 0 & & & & & & 0 & 0 & 0 & & & & I & 0 & 0 & 0 & 0 & \\
\hline $\mathrm{r}_{1}$ & $\mathrm{~F}$ & & & & & & & & & & & & & & & & & & & & & \\
\hline $\mathrm{r}_{2}$ & $-F$ & & & & & & & & & & & & & & & & & & & & & \\
\hline$v_{6}$ & & 0 & 0 & & I & & & & & & & & & & & & & & & & & \\
\hline$v_{7}$ & & I & 0 & & & & & & & & & & & & & & & & & & & \\
\hline $\mathrm{p}_{2}$ & & I & 0 & 0 & 0 & & & & & & & & & & & & & & & & & \\
\hline $\mathrm{P}_{3}$ & & 0 & 0 & $-\mathrm{I}$ & 0 & & & & & & & & & & & & & & & & & \\
\hline $\mathrm{P}_{4}$ & & 0 & 0 & I & 0 & & & & & & & & & & & & & & & & & \\
\hline $\mathrm{p}_{5}$ & & 0 & $-I$ & 0 & 0 & & & & & & & & & & & & & & & & & \\
\hline $\mathrm{x}_{2}$ & & 0 & 0 & & & & & & & & $\mathrm{r}$ & I & I & & & & 0 & 0 & I & 0 & 0 & \\
\hline$r_{3}$ & & & & & & & & & & $\mathrm{~F}$ & & & & & & & & & & & & \\
\hline$r_{4}$ & & & & & & & & & & $-F$ & & & & & & & & & & & & \\
\hline $\mathrm{r}_{5}$ & & & & & & & & & & $-F$ & & & & & & & & & & & & \\
\hline${ }^{P_{6}}$ & & & & & & & & & & & I & 0 & 0 & & & & & & & & & \\
\hline$p_{7}$ & & & & & & & & & & & 0 & I & 0 & & & & & & & & & \\
\hline $\mathrm{P}_{8}$ & & & & & & & & & & & 0 & 0 & I & & & & & & & & & \\
\hline$v_{1}$ & & 0 & 0 & & & & & & & & 0 & 0 & 0 & & & & & I & 0 & 0 & $-I$ & \\
\hline$v_{2}$ & & 0 & 0 & & & & & & & & $\mathrm{~T}$ & 0 & 0 & & & & & & 0 & $-I$ & 0 & \\
\hline$v_{3}$ & & 0 & 0 & & & & & & & & 0 & 0 & 0 & & & & I & 0 & & 0 & 0 & \\
\hline$v_{4}$ & & 0 & 0 & & & & & & & & 0 & I & 0 & & & & 0 & & 0 & & 0 & \\
\hline$v_{5}$ & & I & 0 & & & & & & & & 0 & 0 & 0 & & & & & 0 & 0 & 0 & & \\
\hline$P_{1}$ & & & & & & & & & & & & & & & & & 0 & 0 & I & 0 & 0 & \\
\hline
\end{tabular}

Fig. 21(a). Complete interaction matrix for residential community model.

existence of a perception delay between the attractiveness and in-migration variables. An increase (decrease) in attractiveness of the area will be perceived with delay by the people outside the community. Adding this delay to the Dynamo flow diagram of Fig. 21 essentially completes the Dynamo flow diagram component of the computer-aided model formulation exercise.

\section{SUMMARY AND CONCLUSION}

In this paper an innovative approach to the development of continuous simulations is described. Fundamentally, the new approach is premised upon the presumption that, through computer assistance, the procedure for simulation model formulation can be expedited, systematized, and operationalized. The computer-aided approach is derived from the assertions of causality implicit within Forrester's system dynamics. If implemented, the approach will likely motivate and facilitate the determination of the important sectors, the important quantities to be included, the existence of couplings between the quantities, as well as the identities of both couplings and quantities. A systematic formulation process may produce models which would enjoy a greater degree of credibility and validity. Equally important, such models could be developed quickly.
Computer-internalized models could also be analyzed and tested for stability, reachability, observability and controllability by means of algorithms. All these platitudes remain to be proven in empirical model formulation exercises utilizing computer-assistance, however.

A computer-aided approach could be thought of as comprising three major tasks: (1) development of the Dynamo flow diagram, (2) composition of the equations from the flow diagram, and (3) execution of the simulation model. This paper has concentrated upon task (1); for a brief description of tasks (2) and (3), see [4]. In this context, the use of interaction matrices was explored as a possible data structure for the approach. By use of the interaction matrix, it is possible to infer many of the interactions as nonexistent because of their incompatibility with the rules of system dynamics. Thus the interaction matrix focuses user attention upon just those interactions which are consistent with the notions of system dynamics and forces construction of a model that is rigorously adherent with such notions as a first cut. It was discovered that by consideration of every feasible interaction in the interaction matrix a systematic process for preventing errors of omission to occur results. It was also discovered that roughly $5 / 6$ of the interaction matrix can be filled with inferred zeros 


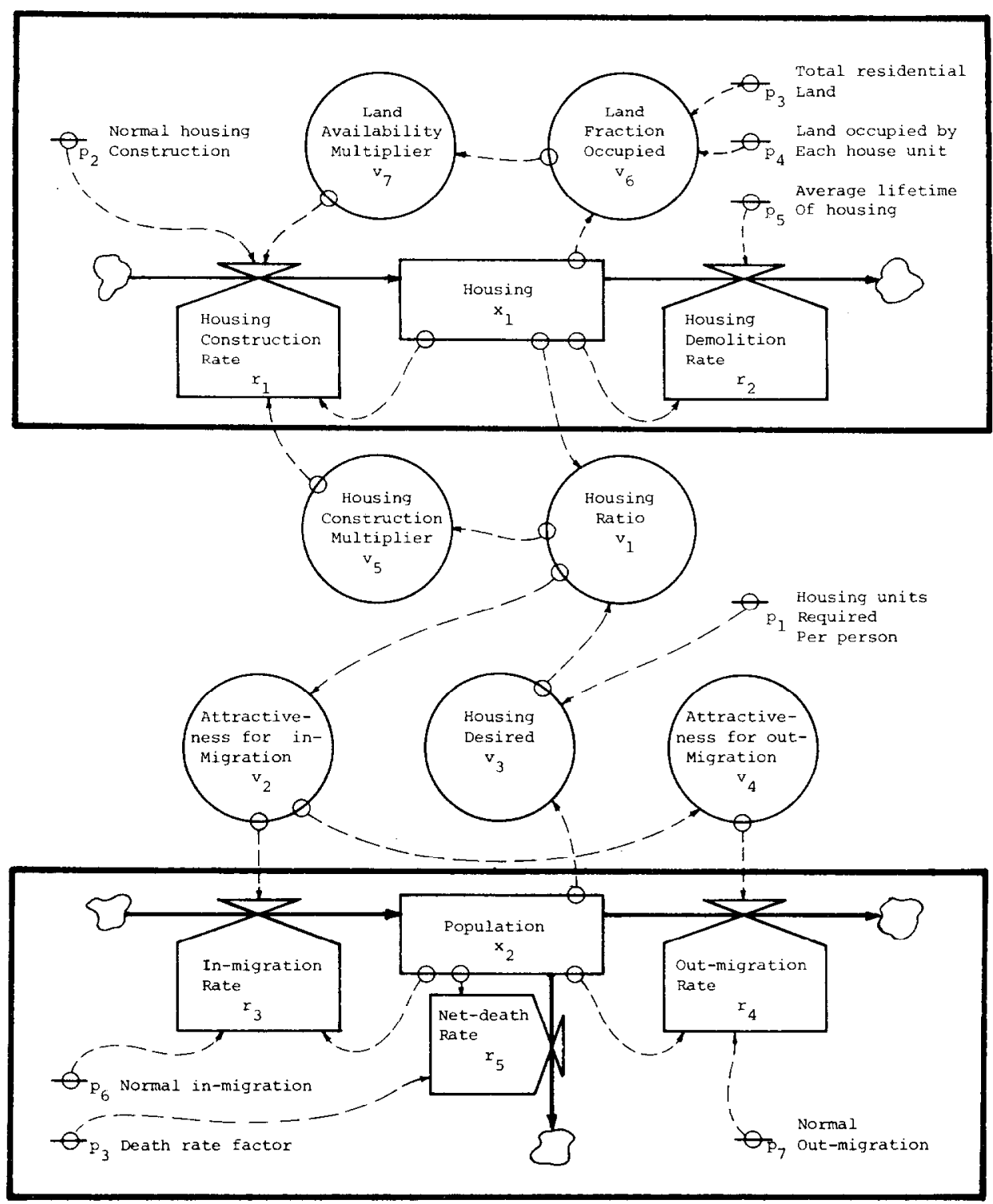

Fig. 21(b). Dynamo flow diagram for completed residential community model.

(blanks), thereby preventing errors of commission to occur in which a link which is incompatible with the rules of system dynamics is inserted.

An overview algorithm was thereafter presented and discussed. The approach was then illustrated through recourse to a classical textbook example-the residential housing model-found in [11]. The approach was found to produce a model that was consistent with the classical textbook example. In this example the specific form of queries posed by software already available $[20,21]$ was indicated. The procedure and queries were both found to be quite compatible with the concerns of modelers who employ system dynamics methodology.

Acknowledgement-The authors are indebted to Mr. H. W. Beights who translated sketchy flow charts and theoretical developments into a formal algorithm and associated software.

\section{REFERENCES}

1. G. Fromm, W. Hamilton and D. Hamilton, Federally Supported Mathematical Models: Survey and Aralysis, National Science
Foundation-RANN publication number NSF-RA-S-74-029 (available from U.S. Government Printing Office. Washington, D.C. 20402, \#038-000-00221-0), 1974.

2. Ways to Improve Management of Federally Funded Computerized Models, National Bureau of Standards, Number LCD75-111. 1975.

3. J. R. Burns, A preliminary approach to automating the process of simulation model synthesis. Proc. of the 7 th Ann. Pittsburgh Conf. on Modeling and Simulation, p. 820 (1976).

4. J. R. Burns, Converting signed digraphs to Forrester schematics and converting Forrester schematics to differential equations. IEEE Trans. on Systems, Man and Cybermetics, SMC-7 (1977)

5. R. Fitz and D. Hornbach, A participative methodology for designing dynamic models through structural models. Proc. of the 7th Ann. Pittsburgh Conf. on Modeling and Simulation, p. 1168 (1976).

6. J. W. Forrester, Industrial Dynamics. M.I.T. Press, Cambridge (1961).

7. J. W. Forrester, Principles of Systems. Wright-Allen Press, Cambridge (1\%8).

8. J. W. Forrester, Urban Dynamics. M.I.T. Press, Cambridge (1969). 
9. J. W. Forrester, World Dynamics. Wright-Allen Press, Cambridge (1977).

10. N. B. Forrester, The Life-Cycle of Economic Development. Wright-Allen Press, Cambridge (1973).

11. M. R. Goodman, Study Notes in System Dynamics. WrightAllen Press, Cambridge (1974).

12. F. Harary, R. Norman and D. Cartwright, Structural Models: An Introduction to the Theory of Directed Graphs. Wiley, New York (1965).

13. J. Kane, A primer for a new cross impact language-KSIM. Technological Forecasting and Social Change 4, 129 (1972).

14. G. J. Klir, An Approach to General Systems Theory. Van Nostrand, New York (1969).

15. R. H. Moll and C. M. Woodside, TR No. S.E. 76-1, Systems Engincering Department, Carleton University, Toronto, Canada (1976)
16. A. Thesen, Some notes on systems models and modeling. Int. J. Systems Sci. 5, 145 (1974).

17. W. Wakeland, A low-budget heuristic approach to modeling and forecasting. Technological Forecasting and Social Change 9 (1976).

18. J. Warfield, Societal Systems. Wiley, New York (1976).

19. J. R. Burns and O. Ulgen, A sector approach to the formulation of system dynamics models. Int. I. Systems Sci. (1978)

20. H. W. Beights, Computer Aided Simulation Methodology, CASM1, User's Guide, Tech. Rep. QT-111-78, Department of Industrial Engineering, Texas Tech University, Lubbock, Texas, 1978.

21. H. W. Beights, Computer Aided Simulation Methodology, CASM1, Programmer's Guide, Tech. Rep. QT-112-78, Department of Industrial Engineering. Texas Tech University, Lubbock, Texas, 1978. 